\title{
Glycemic variation in uncontrolled Graves' disease patients with normal glucose metabolism: Assessment by continuous glucose monitoring
}

\author{
$\mathrm{Gu} \mathrm{Gao}^{1,2} \cdot$ Feng-fei $\mathrm{Li}^{1} \cdot$ Yun Hu${ }^{1} \cdot$ Reng-na Yan ${ }^{1} \cdot$ Bing-li Liu $^{1} \cdot$ Xiao-mei Liu ${ }^{1} \cdot$ Xiao-fei Su${ }^{1} \cdot$ Jian-hua Ma ${ }^{1} \cdot \mathrm{Gang} \mathrm{Hu}^{2}$
}

Received: 29 May 2018 / Accepted: 12 November 2018 / Published online: 4 December 2018

(c) The Author(s) 2018

\begin{abstract}
Purpose To observe the glycemic variation (GV) in uncontrolled Graves' disease (GD) patients with normal glucose metabolism measured by continuous glucose monitoring (CGM).

Methods This was a single-center, open-label, observational study. From January 2017 to October 2017, 20 GD patients with normal glucose metabolism and 24 healthy control subjects were recruited. Serum samples were obtained at 0,30 , and 120 min after oral glucose loading for glucose, insulin, and C-peptide level measurements. Fasting plasma fasting free triiodothyronine (FT3), free thyroxin (FT4), and thyroid stimulating hormone concentrations were also detected. All participants were subjected to a 3-day CGM after baseline data were collected. The primary endpoint was the difference in the mean amplitude of the glycemic excursions between the two groups.

Results Compared with the healthy subjects, the GD patients had higher mean amplitude of glycemic excursions (MAGE) $(P<0.01)$. Multiple linear stepwise regression analysis showed that FT4 level was an independent factor for the MAGE. Interestingly, the GD patients had a significant prolongation in the time to peak glucose, especially after breakfast $(P<0.01)$, and the elevation in the incremental area under the curve of glucose after breakfast till 4 hours later.

Conclusions Uncontrolled GD patients with normal glucose metabolism had a greater GV, and the FT4 level may contributed to the increased GV.
\end{abstract}

Keywords Graves' disease $\cdot$ Glycemic variation $\cdot$ Continuous glucose monitoring $\cdot$ Mean amplitude of glycemic excursions

\section{Introduction}

Impaired glucose tolerance, insulin resistance, and increased insulin secretion were found in patients with hyperthyroidism [1-5]. Known factors, responsible for the

The three authors contributed equally: Gu Gao, Feng-fei Li, and Yun $\mathrm{Hu}$.

Jian-hua Ma

majianhua196503@126.com

$\triangle$ Gang Hu

ghu@njmu.edu.cn

1 Department of Endocrinology, Nanjing First Hospital, Nanjing Medical University, Nanjing, China

2 Jiangsu Key Laboratory of Neurodegeneration, Department of Pharmacology, Nanjing Medical University, Nanjing, China abnormal glucose tolerance, including aberrant metabolic rate, increased endogenous gluconeogenesis, excessive insulin secretion, enhanced glucose absorption, and dawn phenomenon, which are affected by the thyroid hormones [6-8].

Using the oral glucose tolerance test (OGTT) criteria screening newly diagnosed Graves' disease (GD) patients, the incidence of pre-diabetes and diabetes were $41.3 \%$ and $11.3 \%$, respectively, which were higher than those of using glycosylated hemoglobin $\left(\mathrm{HbA}_{1 \mathrm{c}}\right)$ criteria [9]. Continuous glucose monitoring (CGM) provides 288 glucose signals throughout a period of $24 \mathrm{hrs}$. Mean amplitude of glycemic excursions (MAGE), and standard deviation (SD) obtained from CGM were used to assess the daily glycemic variations (GVs) $[10,11]$. Studies demonstrated that GD patients with type 2 diabetes (T2D) had increased fasting and postprandial blood glucose monitored by CGM, and the glucose profile was improved along with the normalization of thyroid function [12], which indicated that the thyroid 
function might be a risk factor of GV. However, the GV in GD patients with a normal glucose metabolism was not well clarified perhaps because of scarcity of studies using CGM. We then performed a single-center, open, and observational study. In this study, we compared the $24 \mathrm{hrs}$ GVs using CGM between GD patients with normal glucose tolerance (NGT) and healthy control subjects.

\section{Materials and methods}

The study was performed in the Department of Endocrinology, Nanjing First Hospital, Nanjing Medical University, from January 2017 to October 2017. The research protocol had passed the approval of the hospital ethics committee on 09 September 2016. Informed consent was obtained from all individual participants included in the study. A total of 33 GD patients and 44 age-gender matched healthy control subjects were enrolled.

Uncontrolled GD patients with NGT were enrolled. Patients were excluded if they had a history of diabetes mellitus or abnormal glucose tolerance, or they had severe complications such as hyperthyroid heart disease, hyperthyroidism crisis, or they had severely impaired liver and kidney function, anemia and psychiatric disorders, or they were pregnant or planning to become pregnant, or they had received systemic hormone therapy within 3 months, or those with acute infection or stress in the previous 4 weeks, or they had abuse of alcohol or drugs.

All the recruited GD patients were admitted as inpatients. OGTT using $75 \mathrm{~g}$ of glucose (dissolved in $200 \mathrm{ml}$ water) was performed at baseline. Serum samples were obtained at 0, 30, and $120 \mathrm{~min}$ after oral glucose loading for blood glucose, insulin, C-peptide level measurements. C-peptide, insulin, and glucose concentrations were measured centrally at the central laboratory in Nanjing First Hospital, Nanjing Medical University. Fasting free triiodothyronine (FT3), free thyroxin (FT4), and thyroid stimulating hormone (TSH) were detected by chemiluminescence (Abbott Laboratories). The normal value of FT4, FT3, and TSH used in our hospital were $9.0-19.0 \mathrm{pmol} / \mathrm{L}, 2.63-5.7 \mathrm{pmol} /$ $\mathrm{L}$, and TSH $0.35-4.94 \mathrm{IU} / \mathrm{mL}$, respectively. $\mathrm{HbA}_{1 \mathrm{c}}$ was measured by a DiaSTAT $\mathrm{HbA}_{1 \mathrm{c}}$ analyzer (Bio-Rad, Hercules, CA).

After OGTT was performed, all the participants in both groups were subjected to a 3-day retrospective CGM (Sofsensor, CGMS-Gold, Medtronic Incorporated, Northridge, USA) in the hospital by the specialist nurse. All the subjects were instructed to maintain usual physical activity and received meals consisting of a total daily caloric intake of $25 \mathrm{kcal} / \mathrm{kg} /$ day $(60 \%$ carbohydrate, $20-25 \%$ lipid, and 15-20\% protein, respectively), and all the subjects were instructed to have breakfast, lunch and dinner at 0700 ,
1100, and 1700, respectively, during the 3 days of CGM period. The CGM was calibrated four times per day by the finger-stick blood glucose concentrations.

The primary endpoint was the difference in MAGE between the two groups. The MAGE was calculated manually for each patient by measuring the arithmetic mean of the ascending and descending excursions between consecutive peaks and nadirs for the same 24-hrs period, and only absolute excursion values $>1 \mathrm{SD}$ were considered as described previously $[13,14]$. The differences in terms of 24-hrs mean blood glucose (MBG), SD, coefficient of variation $(\mathrm{CV} \%)$, the incremental area under curve (AUC) and the time spent in glucose $>7.8,>10.0$, and $<3.9 \mathrm{mmol} / \mathrm{L}$, the hourly MBG, homeostasis model assessment for insulin resistance (HOMA-IR) (fasting plasma glucose (FPG) $\times$ fasting insulin/22.5), and homeostasis model assessment for beta cell function (HOMA- $\beta) \quad($ FINS $\times 20 /(\mathrm{FPG}-3.5))$ between the two groups were also recorded. Hypoglycemia was defined as glucose concentration $<3.9 \mathrm{mmol} / \mathrm{L}$ monitored by CGM. The detailed study protocol was described in the study flow chart (Table 1).

The study was registered with ClinicalTrials.gov identifier: ChiCTR-ROC-17010362.

\section{Statistical methods}

Data were analyzed with the SPSS PASW Statistics 22 Package. Shapiro-Wilk test was used to assess the distribution of data. Normally distributed and continuous variables were presented as mean $\pm \mathrm{SD}$. Non-normal distributed variables were presented as interquartile range). The Mann-Whitney $U$ test was used to compare nonnormal distribution of data. An independent samples $t$ test was used to compare the differences between the two groups. General linear model for repeated measurements was used in the comparison of groups. Bonferroni correction was followed. We used correlation coefficients and multiple linear regression analyses to examine the interrelationships among the GVs and thyroid hormones. All comparisons were two-sided at the 5\% significance level. $P$ value $<0.05$ was considered to be statistically significant.

\section{Results}

\section{Baseline characteristics}

Between January 2017 and October 2017, a total of 33 GD patients and 44 healthy control subjects were recruited for the study. Thirty-three patients were excluded from this study: 13 were from GD group and 20 from control group. They were either owing to abnormal glucose tolerance, or 
Table 1 Study flow chart

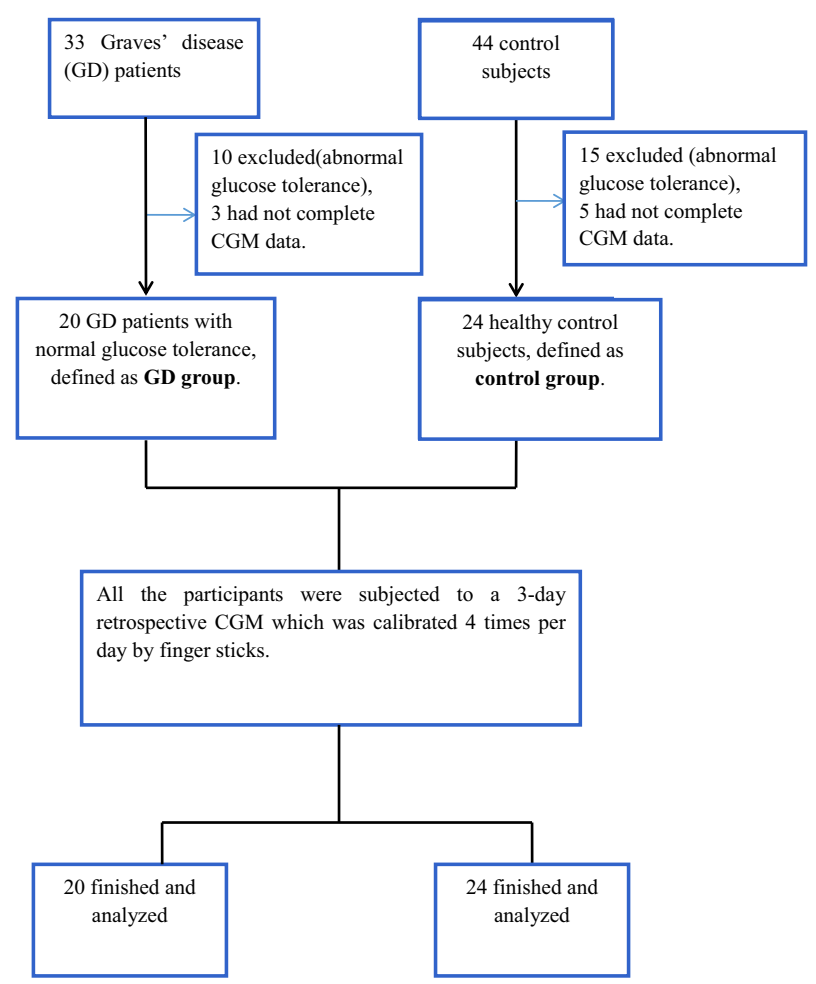

Table 2 Baseline for the study subjects in the two groups

\begin{tabular}{llll}
\hline Items & GD group & Control group & $P$ \\
\hline$N$ & 20 & 24 & $/$ \\
Sex & Male 8, female 12 & Male 10, female 14 & 0.87 \\
Age (year) & $39.8 \pm 13.6$ & $40.5 \pm 12.7$ & 0.84 \\
BMI $\left(\mathrm{kg} / \mathrm{m}^{2}\right)$ & $20.7 \pm 3.0$ & $21.7 \pm 5.5$ & 0.46 \\
$\mathrm{HbA}_{1 \mathrm{c}}(\%)$ & $5.2 \pm 0.3$ & $5.2 \pm 0.3$ & 0.60 \\
TSH $(\mathrm{mIU} / \mathrm{L}))$ & $0.01 \pm 0.00$ & $1.68 \pm 0.74$ & 0.00 \\
FT3 (pmol/L) & $26.53 \pm 12.51$ & $4.54 \pm 0.46$ & 0.00 \\
FT4 (pmol/L) & $45.43 \pm 14.09$ & $13.49 \pm 1.21$ & 0.00 \\
HOMA-IR & $2.1 \pm 1.1$ & $1.9 \pm 0.9$ & 0.54 \\
HOMA- $\beta$ & $84.1 \pm 44.4$ & $75.6 \pm 34.1$ & 0.47 \\
\hline
\end{tabular}

$N$ number, $B M I$ body mass index, $H b A_{l c}$ glycosylated hemoglobin, TSH thyroid-stimulating hormone, FT3 free triiodothyronine, FT4 free thyroxine, HOMA-IR homeostasis model assessment for insulin resistance, HOMA- $\beta$ homeostasis model assessment for beta-cell function

had not completed the CGM data. Thus, there were 20 GD patients with NGT (8 men and 12 women) in the GD group, and 24 healthy control subjects (10 men and 14 women) in the control group (Table 2).
There were no significant demographic differences in age, sex, body mass index and $\mathrm{HbA}_{1 \mathrm{c}}$ between the two groups at baseline (Table 2). OGTT data showed that the blood glucose levels of 30 and 120 min after glucose loading in GD patients were statistically significantly higher than those in the healthy control subjects $(9.8 \pm 1.5$ vs. $8.6 \pm 1.1 \mathrm{mmol} / \mathrm{L}, 7.0 \pm 0.6$ vs. $6.1 \pm 1.2 \mathrm{mmol} / \mathrm{L}, P<0.01$, respectively). Moreover, the FPG levels between the two groups were similar $(5.5 \pm 0.3$ vs. $5.5 \pm 0.3 \mathrm{mmol} / \mathrm{L}, P>$ $0.05)$ (Fig. 1a). In addition, there were no significant differences either in fasting and postprandial C-peptide or insulin concentrations (Fig. 1b, c), or in HOMA-IR and HOMA- $\beta$ between the two groups $(P>0.05$, respectively) (Table 2).

\section{GV profiles}

CGM data showed that the 24-hrs MAGE, MBG, SD, and CV\% were significantly increased in GD group compared with those in the control group $(2.0 \pm 1.2$ vs. $1.2 \pm$ $0.5 \mathrm{mmol} / \mathrm{L}, 6.2 \pm 0.5$ vs. $5.4 \pm 0.6 \mathrm{mmol} / \mathrm{L}, 0.8 \pm 0.4$ vs. $0.5 \pm 0.2 \mathrm{mmol} / \mathrm{L}, P<0.01$, respectively, and $0.1 \pm 0.1 \mathrm{vs}$. $0.1 \pm 0.0, P<0.05)$. In addition, there were significant differences in the hourly MBG between the two groups (all $P<0.05$ ) with exception of 0200 and 0300 (Fig. 2). 

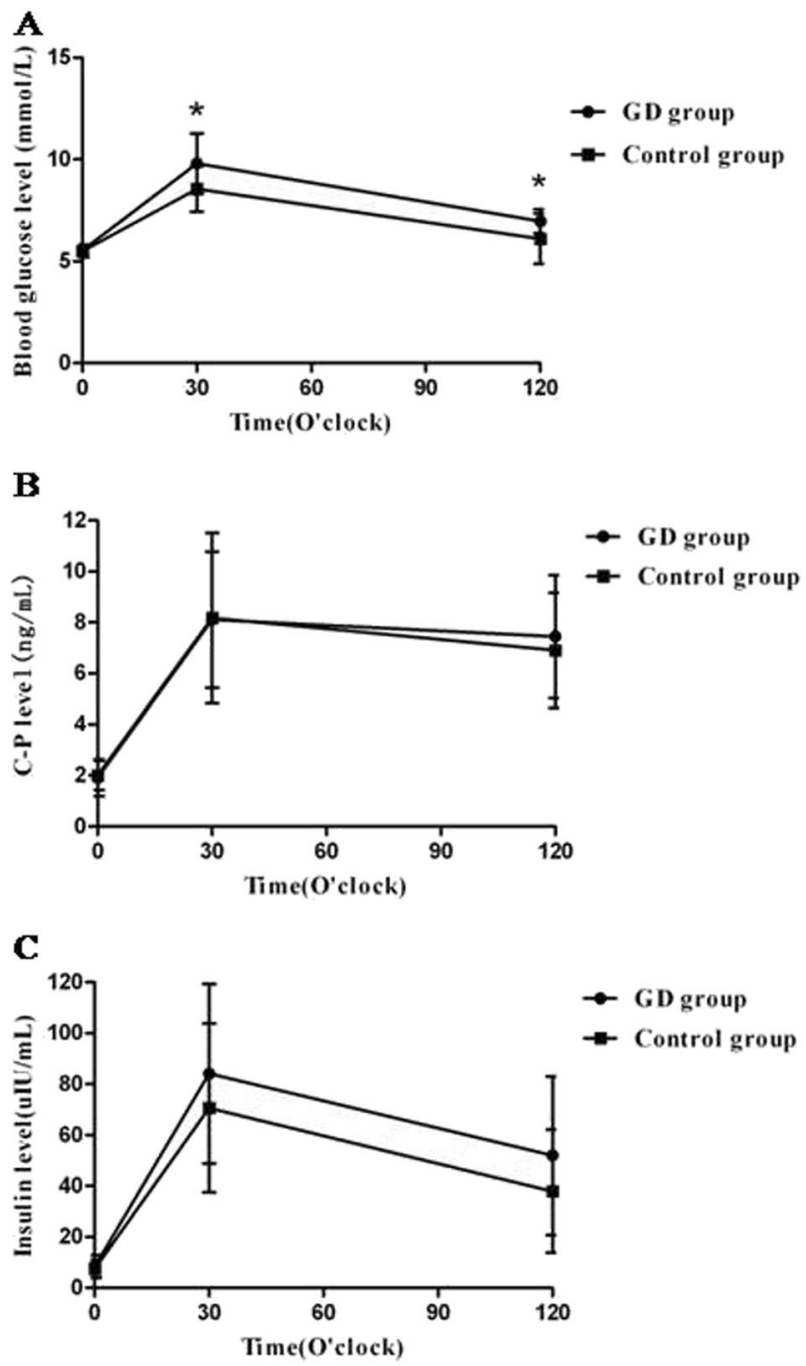

Fig. 1 The blood glucose a, C-peptide b, and insulin levels $\mathbf{c}$ at 0, 30, and $120 \mathrm{~min}$ after glucose loading in the two groups

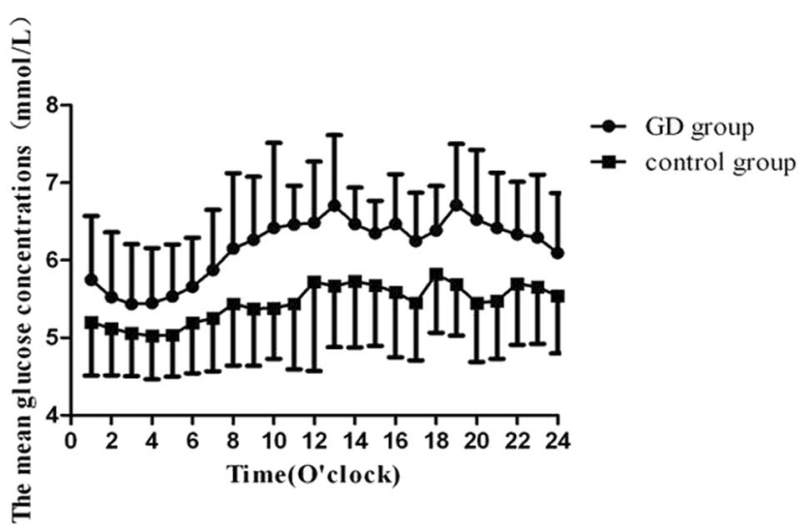

Fig. 2 The average glucose concentrations per hour in the study subjects

Although GD patients had higher peak glucose concentrations after three meals (breakfast: $7.6 \pm 1.3$ vs. $6.1 \pm$

$0.9 \mathrm{mmol} / \mathrm{L}, P<0.01$, lunch: $7.9 \pm 1.2$ vs. $6.3 \pm 1.0 \mathrm{mmol} / \mathrm{L}$, $P<0.01$, dinner: $7.5 \pm 1.0$ vs. $6.3 \pm 1.0 \mathrm{mmol} / \mathrm{L}, P<0.01$ ). They exhibited significant prolongation in the time to peak glucose after breakfast and dinner $(128.0 \pm 60.8$ vs. $81.0 \pm$ $16.9 \mathrm{~min}, \quad 115.0 \pm 43.0$ vs. $80.6 \pm 23.3 \mathrm{~min}, \quad P<0.01$, respectively) compared with healthy control subjects. Of significant importance, we observed that the subjects in GD group had dramatically increased glucose levels from 0400.

In this study, all the subjects got up around 0600, had breakfast, lunch at 0700 and 1100 , respectively. We also analyzed the GV profiles from 0700 to 1100 between the two groups. Although there were no differences in the incremental AUC of $2 \mathrm{hrs}$ postprandial after three meals between the two groups, GD patients showed gradually elevated the incremental AUC of glucose after breakfast till 4 h (1hrs: $18.8 \pm 8.8$ vs. 2 hrs: $49.3 \pm 18.1$ vs. 3 hrs: $88.1 \pm$ 21.7 vs. $4 \mathrm{hrs:} \quad 139.0 \pm 29.0 \mathrm{mmol} / \mathrm{L} /$ day, $\quad P<0.05$, respectively).

The incremental AUC above $7.8 \mathrm{mmol} / \mathrm{L}(0.0(0.0,0.0)$ vs. $0.0(0.0,0.0)), 10.0 \mathrm{mmol} / \mathrm{L}(0.0(0.0,0.0)$ vs. $0.0(0.0$, $0.0)$ ) and below $3.9 \mathrm{mmol} / \mathrm{L}(0.0(0.0,0.0)$ vs. $0.0(0.0,0.0))$ were similar in subjects between the two groups $(P>0.05$, respectively). In addition, the GD patients spent significant more time in glucose concentrations above $7.8 \mathrm{mmol} / \mathrm{L}$ than those of healthy control subjects $(25.0(0.0,125.0) \mathrm{min}$ vs. $0.0(0.0,0.0) \mathrm{min}, P<0.01)$. We did not observe any difference in time spent in glucose concentrations above $10.0 \mathrm{mmol} / \mathrm{L}(0.0(0.0,0.0) \mathrm{min}$ vs. $0.0(0.0,0.0) \mathrm{min}, P>$ $0.05)$ or below $3.9 \mathrm{mmol} / \mathrm{L}(0.0(0.0,0.0) \mathrm{min}$ vs. $0.0(0.0$, $0.0)$ min, $P>0.05$ ) in subjects between the two groups.

\section{Correlation analysis}

We analyzed the correlation of the FT3 and the FT4 levels with GVs and other parameters. On logistic analysis, plasma FT3 level was positively correlated with MBG, SD, MAGE, CV\%, Glu 30, and INS $120(r=0.53, P<0.01 ; r=0.54, P$ $<0.01 ; r=0.46, P<0.01 ; r=0.43, P<0.01 ; r=0.36, P<$ $0.05 ; r=0.35, P<0.05$, respectively). Plasma FT4 level was positively correlated with MBG, SD, MAGE, CV\%, Glu 30, and Glu 120 ( $r=0.49, P<0.01 ; r=0.49, P<0.01$; $r=0.51, P<0.01 ; r=0.39, P<0.01 ; r=0.40, P<0.01$; $r=0.32, P<0.05$, respectively), and negatively correlated with BMI $(r=-0.36, P<0.05)$.

We have performed the statistical correlation of thyroid hormones with HOMA-IR and HOMA- $\beta$. However, we did not observe either plasma FT3 or FT4 level correlated with HOMA-IR $(r=0.25, P>0.05 ; r=0.02, P>0.05$, respectively) and HOMA- $\beta(r=0.24, P>0.05 ; r=-0.00, P>$ 0.05 , respectively).

Multiple linear stepwise regression analysis showed that plasma FT4 level was an independent factor for MAGE $($ Beta $=0.50, P<0.01)$. 


\section{Discussion}

In this study, we found that GD patients with NGT had an increase in GVs in terms of MAGE and SD compared with those of healthy control subjects.

Patients with hyperthyroidism had a much higher rate of developing abnormal glucose tolerance and diabetes mellitus than the general population using OGTT or $\mathrm{HbA}_{1 \mathrm{c}}$ diagnostic criteria $[9,15,16]$. In this study, we used OGTT to evaluate abnormal glucose tolerance in the GD patients instead of $\mathrm{HbA}_{1 \mathrm{c}}$, which may avoid the underestimated prevalence of diabetes in the GD patients caused by the alteration of erythrocytes in circulation [17, 18].

Using the CGM data, the GD patients with T2D exhibited not only elevated postprandial hyperglycemia, but also fasting hyperglycemia which might be caused by the dawn phenomenon [12]. Moreover, studies indicated that thyroid function may be a risk factor for glycemic control in GD patients $[12,19]$. However, the knowledge of GV in GD patients with NGT is limited.

In this study, CGM data showed that GD patients with normal glucose metabolism had increased GVs in terms of MAGE and SD, which was significantly higher than those of healthy control subjects. Of significant importance, we observed that the subjects in GD group had dramatically increased glucose levels from 0400, prolongation in the time to peak glucose, and higher peak glucose concentrations after breakfast. Our findings agreed with the previous study which indicated a significant increase in glucose concentration after 0400 in GD patient with T2D [12]. The mal-rhythm of glucose metabolism in GD patients may be caused by the increased insulin-antagonizing hormone such as growth hormone, insulin resistance, and gluconeogenesis $[20,21]$. However, we did not observe any difference in HOMA-IR or HOMA- $\beta$ between the two groups, which might be the reason that the sample size was relative modest.

Studies indicated that GD patients had excessive insulin secretion in response to increased gluconeogenesis or insulin-antagonizing hormones $[6,22]$. The higher insulin concentrations might contribute to hypoglycemia in GD patients with or without diabetes [23-25]. However, we did not observe any differences in the incremental AUC below $3.9 \mathrm{mmol} / \mathrm{L}$ between the two groups. Future studies are needed to identify whether hypoglycemia is a common symptom in the GD patients.

FT3 and FT4 were identified as risk factors for higher plasma glucose levels [26]. Interestingly, our data indicated that the FT4 levels were positively correlated with MAGE. Although our data are not conclusive, it does give some indication that the thyroid hormone may influence the GVs.

Our study still has other limitations. First, the study only observed the Chinese population, so the situation might not be the same for other populations. Second, we did not observe for a long time period to determine whether GV could be improved after therapy. Third, we had no data clarifying the mal-rhythm of glucose metabolism in GD patients.

In conclusion, GD patients with normal glucose metabolism had greater glycemic fluctuations when compared with those of healthy control subjects. Our data also indicated that the FT4 level may contribute to the increased GV.

Acknowledgements We appreciate professor Frank Elliott who contributed to the manuscript correction and final approval of this manuscript. (204 Pearce Road, Pineville, Louisiana, USA 71360).

Funding This study was funded by the Nanjing Public Health Bureau Project (No. YKK16137), the Science and Technology Support Program of Jiangsu Province (CN) (No. BK20170136 and SBK2015042970), and the National Natural Science Foundation of China (No. 81500606).

Author contributions G.H., JH.M., XF.S., and G.G. contributed to the conception and design of the study. G.G., BL.L., XM.L., Y.H., and RN.Y. contributed to the conduct/data collection. G.G.., RN.Y., and BL.L., contributed to the data analysis. G.G. and FF.L. contributed to writing the manuscript and the final approval of the manuscript.

\section{Compliance with ethical standards}

Ethics approval All procedures performed in studies involving human participants were in accordance with the ethical standards of the institutional research committee and with the 1964 Helsinki declaration and its later amendments or comparable ethical standards.

Informed consent Informed consent was obtained from all individual participants included in the study.

Conflict of interest The authors declare that they have no conflict of interest.

Open Access This article is distributed under the terms of the Creative Commons Attribution 4.0 International License (http://crea tivecommons.org/licenses/by/4.0/), which permits use, duplication, adaptation, distribution, and reproduction in any medium or format, as long as you give appropriate credit to the original author(s) and the source, provide a link to the Creative Commons license, and indicate if changes were made.

\section{References}

1. B. Ahrén, Hyperthyroidism and glucose intolerance. Acta Med. Scand. 220(1), 5-14 (2010)

2. M. Fukuchi, M. Shimabukuro, Y. Shimajiri, Y. Oshiro, M. Higa, H. Akamine, I. Komiya, N. Takasu, Evidence for a deficient pancreatic beta-cell response in a rat model of hyperthyroidism. Life. Sci. 71(9), 1059-1070 (2002)

3. N. Karbalaei, A. Noorafshan, E. Hoshmandi, Impaired glucosestimulated insulin secretion and reduced $\beta$-cell mass in pancreatic islets of hyperthyroid rats. Exp. Physiol. 101(8), 1114-1127 (2016)

4. D. Ozdemir, S. Dagdelen, A. Usman, Serum adiponectin levels and changes in glucose metabolism before and after treatment for 
thyroid dysfunction. Turk. J. Endocrinol. Metab. 17(4), 1849 (2013)

5. E. Maratou, D.J. Hadjidakis, M. Peppa, M. Alevizaki, K. Tsegka, V. Lambadiari, P. Mitrou, E. Boutati, A. Kollias, T. Economopoulos, Studies of insulin resistance in patients with clinical and subclinical hyperthyroidism. Eur. J. Endocrinol. 160(5), 785-790 (2009)

6. G.D. Dimitriadis, S.A. Raptis, Thyroid hormone excess and glucose intolerance. Experimental and clinical endocrinology \& diabetes: official journal, German Society of Endocrinology [and] German. Diabetes Assoc. 109(Suppl 2), S225-S239 (2001)

7. Z.T. Bloomgarden; American Diabetes Association Annual Meeting, 1999: insulin action and the development of type 2 diabetes. Diabetes Care 23(2), 248 (2000)

8. Luo, Y.H., Zhu, M., Wang, D.G., Yang, Y.S., Tan, T., Zhu, H., He, J.F, Yangxin Tongmai formula ameliorates impaired glucose tolerance in children with Graves' disease through upregulation of the insulin receptor levels. Acta Pharmacol. Sin. 39(6), 923-929 (2018)

9. L. Yang, X. Shen, S. Yan, X. Yuan, J. Lu, W. Wei, HbA1c in the diagnosis of diabetes and abnormal glucose tolerance in patients with Graves' hyperthyroidism. Diabetes Res. Clin. Pract. 101(1), 28-34 (2013)

10. Z. Jian, L. Hong, X.W. Ran, W.Y. Yang, L. Qiang, Y.D. Peng, Y. B. Li, G. Xin, X.J. Luan, W.Q. Wang, Reference values for continuous glucose monitoring in Chinese subjects. Diabetes Care 32(7), 1188-1193 (2009)

11. Z. Jian, L. Hong, X. Ran, W. Yang, L. Qiang, Y. Peng, Y. Li, G. Xin, X. Luan, W: Wang, Establishment of normal reference ranges for glycemic variability in Chinese subjects using continuous glucose monitoring. Med. Sci. Monit. Int. Med. J. Exp. Clin. Res. 17(1), CR9-CR13 (2011)

12. K. Torimoto, Y. Okada, T. Arao, H. Mori, S. Yamamoto, M. Narisawa, A. Kurozumi, Y. Tanaka, Glucose variability before and after treatment of a patient with Graves' disease complicated by diabetes mellitus: assessment by continuous glucose monitoring. Endocr. J. 61(4), 321 (2014)

13. F. Li, X. Xu, L. Fu, X. Su, J. Wu, C. Lu, L. Ye, J. Ma, Influence of acarbose on plasma glucose fluctuations in insulin-treated patients with type 2 diabetes: a pilot study. Int. J. Endocrinol. 2015, 903524 (2015)

14. F.F. Li, L.Y. Fu, W.L. Zhang, X.F. Su, J.D. Wu, J. Sun, L. Ye, J. $\mathrm{H}$. Ma, Blood glucose fluctuations in type 2 diabetes patients treated with multiple daily injections. J. Diabetes Res. 2016, 1028945 (2015)

15. J.W. Doar, T.C. Stamp, V. Wynn, T.K. Audhya, Effects of oral and intravenous glucose loading in thyrotoxicosis. Studies of plasma glucose, free fatty acid, plasma insulin and blood pyruvate levels. Diabetes 18(9), 633 (1969)

16. K. Kreines, M. Jett, H.C. Knowles Jr, Observations in hyperthyroidism of abnormal glucose tolerance and other traits related to diabetes mellitus. Diabetes 14(11), 740-744 (1965)

17. S. Anantarapu, S. Vaikkakara, A. Sachan, B.V. Phaneendra, M.M. Suchitra, A.P. Reddy, S. Epuri, A. Mukka, D. Vemvakam, Effects of thyroid hormone replacement on glycated hemoglobin levels in non diabetic subjects with overt hypothyroidism. Arch. Endocrinol. Metab. 59(6), 495 (2015)

18. M. Messarah, M. Saoudi, A. Boumendjel, M.S. Boulakoud, A.E. Feki, Oxidative stress induced by thyroid dysfunction in rat erythrocytes and heart. Environ. Toxicol. Pharmacol. 31(1), 33-41 (2011)

19. Y. Hu, G. Gao, R.N. Yan, F.F. Li, X.F. Su, J.H. Ma, Glucose metabolism before and after radioiodine therapy of a patient with Graves' disease: Assessment by continuous glucose monitoring. Biomed. Rep. 7(2), 183 (2017)

20. A. Iranmanesh, G. Lizarralde, M.L. Johnson, J.D. Veldhuis, Nature of altered growth hormone secretion in hyperthyroidism*. J. Clin. Endocrinol. Metab. 72(1), 108 (1991)

21. L.P. Klieverik, S.F. Janssen, A.V. Riel, E. Foppen, P.H. Bisschop, M.J. Serlie, A. Boelen, M.T. Ackermans, H.P. Sauerwein, E. Fliers, Thyroid hormone modulates glucose production via a sympathetic pathway from the hypothalamic paraventricular nucleus to the liver. Proc. Natl. Acad. Sci. USA 106(14), 5966-5971 (2009)

22. Z. Liang, G.C. Luo, Q.H. Hu, M.Z. Li, N. Chen, D.W. Yan, J. Chai, Z.L. Chen, H.Y. Li, H.T. Ou, Evaluation of insulin secretion and insulin resistance by hyperglycemic clamp in Graves disease patients with impaired glucose tolerance. Zhonghua Nei. Ke. Za. Zhi. 46(7), 559-561 (2007)

23. X. Wang, X.L. Xu, X.L. Zhao, X.W. Ma, H. Yu, H. Gong, S.R. Zhang, F.L. Chen, Hypoglycemia due to insulin binding antibodies in a patient with insulin-treated type 2 diabetes and Graves' disease. Endocrine 43(1), 236-237 (2013)

24. B. Paudyal, M. Shakya, B. Basnyat, Spontaneous hypoglycaemia in a patient with Graves' disease. BMJ Case Rep. 2016, bcr2016214801 (2016)

25. E. Roh, Y.A. Kim, E.J. Ku, J.H. Bae, H.M. Kim, Y.M. Cho, Y.J. Park, K.S. Park, S.Y. Kim, S.H. Kwak, Two cases of methimazole-induced insulin autoimmune syndrome in graves' disease. Endocrinol. Metab. 28(1), 55-60 (2013)

26. D.T. Paul, F.H. Mollah, M.K. Alam, M. Fariduddin, K. Azad, M. I. Arslan, Glycemic status in hyperthyroid subjects. Mymensingh Med. J. 13(1), 71 (2004) 